\title{
The Role of Positron-emission Tomography-Computed Tomography for Prediction of Treatment Response After Chemoradiation for Advanced-stage Head and Neck Cancer
}

\author{
James $\mathrm{P}$ Malone, MD, FACS ${ }^{1}$ Simon Bekker, MD² and $\mathrm{K}$ Thomas Robbins, MD, FRCSC, FACS ${ }^{3}$
}

1. Associate Professor and Residency Program Director; 2. Clinical Instructor; 3. Professor, Division of Otolaryngology - Head and Neck Surgery, and Director, Simmons Cancer Institute, Southern Illinois University school of Medicine

\begin{abstract}
Concurrent chemoradiotherapy (CRT) is a common treatment modality for patients with advanced-stage head and neck cancer and results in high rates of locoregional disease control. Accurate assessment of disease response following completion of treatment is necessary to detect persistent disease and to direct surgical intervention if necessary. ${ }^{18 F-f l u o r o d e o x y g l u c o s e ~(F D G) ~ p o s i t r o n-e m i s s i o n ~ t o m o g r a p h y ~(P E T) ~ o r ~ c o m b i n e d ~}$ PET-computed tomography (CT) has become a valuable tool in the assessment of treatment response after chemoradiotherapy. While the precise timing of the scan remains controversial, the high negative predictive value of post-treatment PET-CT strongly suggests that a complete clinical response has been achieved. The functional imaging information provided by PET-CT can be combined with the structural imaging of contrastenhanced CT or magnetic resonance imaging (MRI) and physical examination to better predict those patients who would benefit from post-treatment surgical intervention.
\end{abstract}

\section{Keywords}

Positron-emission tomography-computed tomography (PET-CT), head and neck cancer, chemoradiation, treatment response

Disclosure: The authors have no conflicts of interest to declare.

Received: March 19, 2011 Accepted: August 9, 2011 Citation: US Oncology \& Hematology, 2011;7(2):103-6 DOI: 10.17925/OHR.2011.07.2.103

Correspondence: James P Malone, MD, Department of Surgery, Southern Illinois University School of Medicine, PO Box 19649, Springfield, IL 62794-9649. E: jmalone@siumed.edu

Chemoradiotherapy (CRT) has become a common treatment approach for patients with advanced head and neck cancer, particularly when the disease is unresectable or the loss of function from surgery is excessive. Following such treatment, the majority of patients show a complete clinical response in the primary site. Some of these patients do not have a complete pathologic response, however, and their subclinical disease is difficult to confirm unless the patient subsequently undergoes surgery. The development of positron-emission tomography-computed tomography (PET-CT) imaging represents an important advance in helping the clinician to determine which patients are most likely to have persistent cancer following CRT. Such information is critical to providing the optimal chance for disease control as this relates both to the tumor in the primary site and the regional lymph nodes.

\section{Radiologic Interpretation of Positron-emission Tomography-Computed Tomography After Chemoradiotherapy}

Combined PET-CT examination of patients after CRT allows the integration of both anatomic and structural information with functional metabolic activity in the assessment of persistent disease. The timing of the assessment is controversial, as metabolic tracer uptake and activity can be appreciated for weeks to months after CRT. Persistent disease, inflammation, superimposed infection, and radionecrosis can all potentially contribute to residual tracer uptake (see Figure 1). Proper interpretation needs to account for such post-treatment changes and appropriately distinguish them from persistent disease.

In a recent review of published literature assessing the utility of PET and combined PET-CT for monitoring patients after CRT, focal tracer uptake was the parameter generally utilized to discriminate between persistent disease and post-treatment changes. ${ }^{1}$ In general, focal asymmetric uptake within the primary site or lymph nodes, with intensity greater than the surrounding background and/or blood pool, should raise suspicion of potentially persistent disease. By contrast, post-treatment changes often appear non-focal and diffuse, conform to radiotherapy target volumes, and typically exhibit mild to moderate tracer uptake intensity.

While much emphasis is placed on the qualitative interpretation and integration of PET-CT, the functional data obtained from PET-CT enables qualitative assessment of focal tracer uptake as well as quantitative analysis of metabolic activity. Despite this, such quantitative information, as measured by standard uptake values (SUVS) alone, may not be able to reliably and consistently differentiate tumor from inflammation. For example, a quantitative SUV threshold of 2.9 was shown by Yao et al. to identify residual nodal disease, but this value was not confirmed in subsequent studies in similar patient populations. ${ }^{2.3}$ 
Figure 1: Post-treatment Positron-emission TomographyComputed Tomography Scan
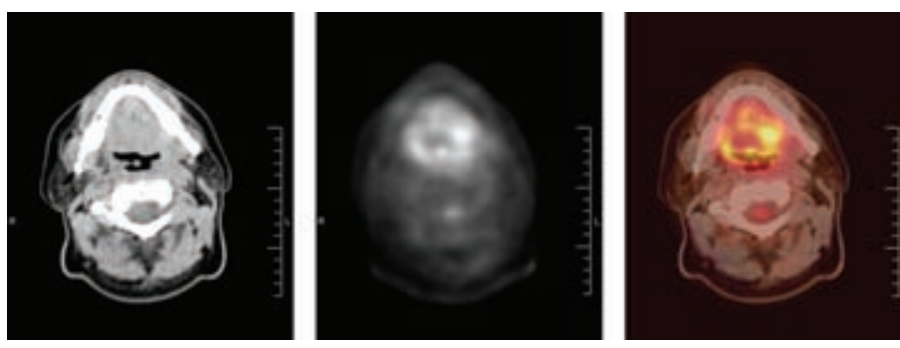

Scans demonstrate residual ${ }^{18} \mathrm{~F}$-fluorodeoxyglucose uptake (standard uptake value 4.6) due to inflammation at the primary tumor site after completion of chemotherapy for squamous cell carcinoma of the left tongue base.

Additional quantitative parameters currently being investigated may broaden the ability to delineate persistent disease as well as partial response. These include SUVLean and SUVLean Peak for more reliable and reproducible assessment of measurable tumor responses. ${ }^{4}$ Likewise, new parameters such as total metabolic tumor volume and burden may provide additional prognostic information..$^{5-8}$

\section{Prediction of Response to Chemoradiotherapy}

An accurate and timely assessment of disease response after completion of CRT is critical for determining the need for surgical salvage versus clinical observation alone in patients with advanced head and neck cancer. As a result of better anatomic localization, combined PET-CT has fewer equivocal results and is more accurate than PET alone for the detection of head and neck cancer. ${ }^{9,10}$

The advantage of PET-CT over traditional imaging modalities, such as CT or magnetic resonance imaging (MRI), is its ability to provide physiologic information about potential regions of tumor metabolic activity in conjunction with anatomic localization. In addition, PET-CT provides easier access to whole-body information about the presence or absence of distant lesions (with lower radiation than $\mathrm{CT}$ and lower cost than MRI) that can affect prognosis and treatment. The ability of PET-CT to accurately predict disease response at the primary tumor site and cervical lymph nodes following CRT may prevent unnecessary surgical interventions designed to determine the completeness of non-operative treatment. This will lead to a potential reduction in patient morbidity and cost of treatment.

\section{Assessment of the Primary Tumor Site}

A limited number of studies have examined the utility of PET-CT specifically for the assessment of tumor response at the primary tumor site after CRT..$^{3,1,12}$ There is substantial variability in the sensitivity and specificity of PET-CT for the detection of local disease based on these studies. The observed variations may be partially due to the time interval between completion of treatment and scanning. Similarly, a higher number of false-positive PET-CT scans are likely to be due to acute or chronic inflammation (see Figure 1). Inflammatory changes at the primary tumor site are typically related to recent treatment resulting in a relatively Iow positive predictive value (PPV) of PET-CT in detecting residual disease.

The negative predictive value (NPV) of PET-CT at the primary tumor site is of much greater utility for confirming disease response. In multiple studies, the NPV of PET-CT for primary tumor control has been shown to be $>90 \% .{ }^{3,11,12}$ A recent study on early (six- to eight-week) post-treatment PET-CT in patients treated with intra-arterial CRT revealed a NPV of $92 \%$ for the primary site. Ong et al. recently reported specificity and NPV of $95 \%$ and $97 \%$ respectively for PET-CT in the assessment of local disease in 65 patients treated with concurrent CRT for advanced squamous cell carcinoma of the head and neck. ${ }^{3}$ Only six patients had abnormal or equivocal [18F]-2-fluoro-2-deoxyD-glucose-PET (18F-FDG-PET) uptake at the primary site at a median time of 12 weeks following treatment. None of these patients had local disease recurrence during the follow-up period. In a study by chen et al. of patients with advanced oropharyngeal cancer treated with CRT, PET-CT performed an average of 7.3 weeks after treatment had a NPV of $95.7 \%$ in predicting residual tumor at the primary tumor site. ${ }^{12}$ Previous studies with PET alone have also been indicative of excellent NPV at the primary tumor site. A recent meta-analysis by Isles et al. using PET alone for detection of disease after radiotherapy or CRT reported an NPV of $95 \% .^{13}$

A negative post-treatment PET-CT at the primary tumor site provides a very accurate and reliable assessment of disease status. Absence of ${ }^{18}$ F-FDG-PET uptake in the primary tumor bed helps to confirm nonspecific abnormalities on CT scan, such as scarring or radiation changes that do not represent residual tumor. Consequently, a negative PET-CT may eliminate the need for evaluation and/or biopsy of the primary site under anesthesia to confirm the absence of disease and avoid the inherent risks of radionecrosis associated with biopsy of irradiated tissue. One can therefore suggest that panendoscopy and biopsy may be deferred in those patients with complete tumor response on clinical examination and a negative PET-CT after the completion of CRT.

\section{Assessment of Cervical Lymph Nodes}

The utility of PET-CT imaging for the assessment of residual neck nodal disease after definitive CRT has been an area of intense study and debate. Historically, in patients presenting with clinically advanced nodal disease (N2 or N3), the conventional approach has been to perform a planned neck dissection after completion of $\mathrm{CRT}$, regardless of the extent of disease response in the cervical nodes. ${ }^{14-16}$ The rationale behind planned neck dissection in this group of patients was to remove any persistent nodal disease that could not be detected on physical examination or structural imaging studies and to confirm a complete pathological response.

Although still controversial, the treatment paradigm of routine planned neck dissection after CRT for patients with advanced nodal disease is beginning to change. Evidence from recent studies suggests that posttreatment neck dissection may not be necessary in patients with advanced neck disease achieving a complete clinical response after CRT based on physical examination and imaging studies that include the use of PET or PET-CT. 3,11,17-19

At present, there have been no randomized clinical trials evaluating the effectiveness of PET or PET-CT for the assessment of nodal response after CRT. Data derived from retrospective studies, however, provide a valuable insight into the utility of this imaging modality for the prediction of disease response and patient management. 
Similar to assessment of the primary tumor site, studies have shown significant variability in the PPV of PET or PET-CT for predicting persistent or recurrent nodal disease with PPV ranging from 18.2 to 91\%. 3 , 11,12,17,20-22 The exact reason for such wide ranges in PPV for the neck in is unclear. It may be related to the lack of consistent timing of posttreatment PET-CT, both within and between different studies, or the use of combined PET-CT versus PET alone.

Conversely, the real value of post-treatment PET-CT rests in its negative findings. In a meta-analysis by Isles et al. for PET alone, the pooled mean NPV for the detection of persistent or recurrent disease was $96 \%{ }^{13}$ In individual studies utilizing PET-CT surveillance after CRT, the NPV ranged from 94 to $100 \%$ in predicting nodal response and corroborating the earlier data from PET alone., , 11,20,23 $^{2}$

The high NPV of PET-CT for detection of persistent nodal disease after CRT is resulting in a significant change in patient management away from routine planned neck dissection and towards a more conservative approach based on clinical and radiologic response to treatment. A variety of algorithms have been proposed for the assessment of patients after completion of CRT that incorporate the use of functional imaging (PET), structural imaging (CT and MRI), and routine physical examination. ${ }^{1-3,24}$ The overall approach of such algorithms includes early (four to six weeks) assessment of clinical response by physical examination.

Imaging utilizing PET-CT and contrast-enhanced CT or MRI scan is obtained six to 12 weeks after treatment. Patients achieving a complete clinical response based on physical examination and imaging studies may be observed (see Figure 2), whereas those patients with progressive disease or palpable and/or PET-positive disease are considered for surgical intervention.

Patient examination, interpretation of radiological studies, and subsequent management are best performed in a multidisciplinary setting. All patients should receive ongoing, routine follow-up for tumor surveillance.

\section{Timing of Positron-emission Tomography- Computed Tomography and the Need for Surgical Intervention}

The appropriate timing of PET-CT after CRT is also an area of ongoing investigation and debate. The optimal time interval between completion of CRT and PET-CT imaging should result in an accurate assessment of disease response in a timely manner. This will direct appropriate surgical intervention, if needed, or allow for conservative clinical observation.

PET-CT scans performed too early after treatment may produce a high rate of false-positive studies due to treatment-related inflammation. Conversely, persistent disease below the level of detection for PET-CT may produce false-negative results if scanning is carried out too early.

A recent study performed by a multidisciplinary head and neck oncology team demonstrated an overall accuracy rate of only $60 \%$ at the primary site for PET-CT performed between six and eight weeks after the completion of CRT. ${ }^{11}$ The NPV for the primary site as well as for the cervical nodes was, however, highly predictive of adequate
Figure 2: Pre- (top) and Post-treatment (bottom) Positronemission Tomography-Computed Tomography Scans

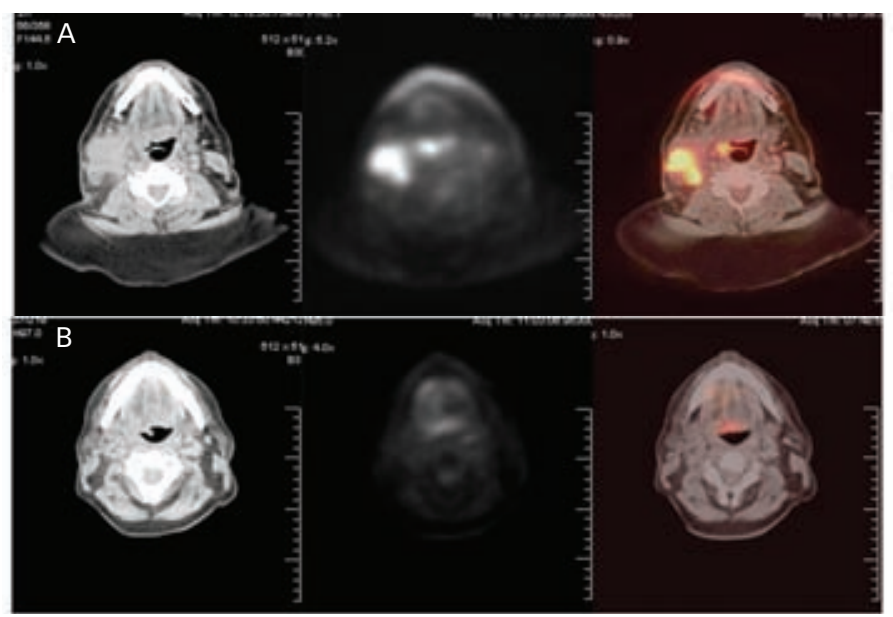

A. Pre-treatment positron-emission tomography-computed tomography (PET-CT) scan demonstrating intense ${ }^{18} \mathrm{~F}$-fluorodeoxyg/ucose $\left({ }^{18} \mathrm{~F}\right.$-FDG) uptake in metastatic right cervical lymph nodes in a patient with squamous cell carcinoma of the right tonsil. B. Post-treatment PET-CT scan with the resolution of ${ }^{18} \mathrm{~F}$-FDG uptake in the right cervical nodes after completion of chemoradiotherapy (CRT) indicative of a complete clinical response.

From left to right: non-contrast CT, PET, PET-CT fusion.

Figure 3: Proposed Management Scheme for Patients Completing Chemoradiotherapy for Advanced Head and Neck Cancer

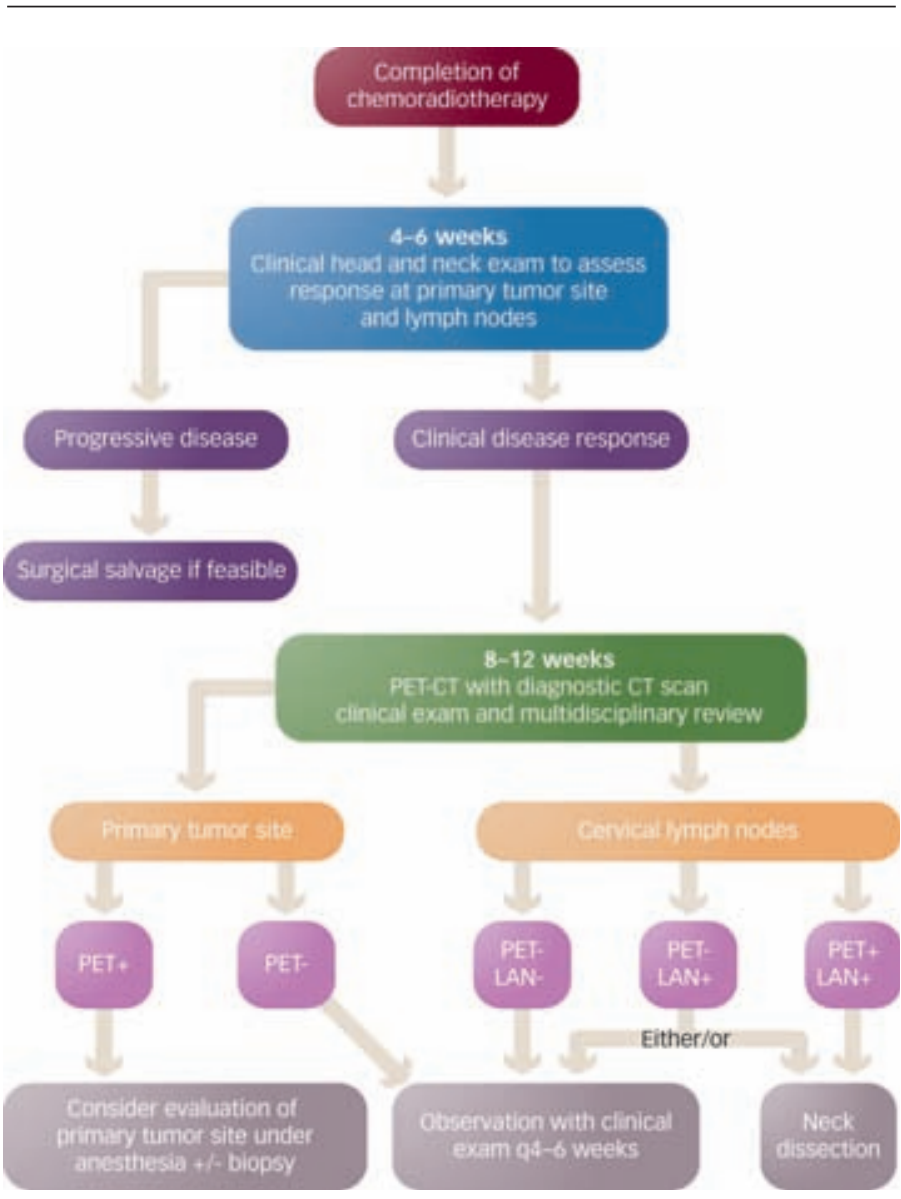

Management scheme utilizes clinical exam and post-treatment imaging. $C T=$ computed tomography; $L A N=$ clinically detectable lymphadenopathy; $P E T+=$ positive positron-emission tomography; PET- = negative positron-emission tomography. 
disease control in this study. A protracted time interval between CRT and surgical intervention may delay the treatment of persistent disease or increase morbidity related to surgery after the onset of fibrosis in the neck.

An initial meta-analysis of the role of PET alone for detecting residual or recurrent head and neck cancer demonstrated greater sensitivity for scans performed 10 or more weeks after treatment. ${ }^{13}$

With the improved accuracy of combined PET-CT over PET alone, recent studies utilizing the combined imaging modality have demonstrated that a negative scan performed within six to eight weeks of completion is highly accurate (NPV 94-100\%) for cervical lymph nodes. ${ }^{11,23}$ Other investigators recommend waiting until approximately 10 to 12 weeks after CRT in order to optimize PET-CT sensitivity and specificity, while still allowing adequate time for surgical intervention before the onset of extensive tissue fibrosis. ${ }^{1,3,13,20}$ Wide variability in the timing of scanning within each of these studies may affect the overall predictive values for post-treatment PET-CT.

The authors recommend an algorithm for patient management after CRT that comprises physical examination four to six weeks after completion of treatment to assess initial disease response, followed shortly thereafter by imaging with contrast-enhanced CT and PET-CT (see Figure 3). In order to prevent an unnecessary delay in identifying and treating residual disease, imaging should be performed no later than 12 weeks after the completion of treatment, with a preference towards earlier imaging (at eight weeks).

If the results of the physical examination or the imaging studies are suggestive of persistent or progressive disease, appropriate surgical intervention should be performed. Ideally, the assessment of treatment response should be performed in a collaborative environment with input from the patient's head and neck surgeon, medical oncologist, radiation oncologist and radiologist. The multidisciplinary setting provides the best context for interpreting the extent of disease response based on clinical and radiologic findings. It also enables the team to determine the direction for further patient management.

\section{Conclusion}

CRT for organ preservation in patients with advanced-stage head and neck cancer produces an excellent locoregional response rate. The addition of functional imaging (PET) to the traditional structural imaging methods (physical exam, CT, and MRI) has substantially improved the management of patients with advanced-stage head and neck cancer after CRT. It has also reduced the morbidity associated with routine surgical intervention regardless of clinical response.

The use of PET-CT with its high NPV has become a reliable tool for helping to determine which patients have achieved a complete tumor response at the primary tumor site and cervical lymph nodes. Patients with a negative post-treatment PET-CT and no evidence of disease on physical examination should be considered for observation rather than planned neck dissection.

There is some disagreement among investigators as to the exact timing of PET-CT after the completion of CRT. Although early PET-CT (after six to eight weeks) maintains a high NPV, there is a higher likelihood of false-positive findings and thus a lower PPV than scans performed longer (12 weeks) after treatment.

Ultimately, clinicians must strive to achieve a balance between timely and accurate assessments of tumor response and preventing the risks associated with delaying the treatment of persistent disease.
1. Schöder $\mathrm{H}$, Fury $\mathrm{M}$, Lee $\mathrm{N}$, et al., PET monitoring of therapy response in head and neck squamous cell carcinoma, I Nucl Med, 2009;50:74S-88S

2. Yao M, Smith RB, Graham MM, et al., The role of FDG PET in management of neck metastasis from head-and-neck cancer after definitive radiation treatment, Int J Radiat Oncol Biol Phys, 2005;63:991-9.

3. Ong SC, Schöder $\mathrm{H}$, Lee NY, et al., Clinical utility of 18F-FDG PET/CT in assessing the neck after concurrent chemoradiotherapy for locoregional advanced head and neck cancer, I Nucl Med, 2008;49:532-40.

4. Wahl R, Jacene H, Kasamon $\mathrm{Y}$, et al., From RECIST to PERCIST: Evolving considerations for PET response criteria in solid tumors, I Nucl Med, 2009:50:122S-50S.

5. Baek C, Chung MK, Son YI, et al., Tumor volume assessment by 18F-FDG PET/CT in patients with oral cavity cancer with dental artifacts on CT and MR images, J Nucl Med, 2008;49:1422-8.

6. Seol Y, Kwon BR, Song MK, et al., Measurement of tumor volume by PET to evaluate prognosis in patients with head and neck cancer treated by chemoradiation therapy, Acta Oncol 2010:49:201-8.

7. La T, Filion EJ, Turnbull BB, et al., Metabolic tumor volume predicts for recurrence and death in head-and-neck cancer, Int J Radiat Oncol Biol Phys, 2009;74:1335-41.

8. Chung M, Jeong HS, Son YI, et al., Metabolic tumor volumes by [18F]-fluorodeoxyglucose PET/CT correlate with occult metastasis in oral squamous cell carcinoma of the tongue, Ann Surg Oncol, 2009;16:3111-7.

9. Schöder $\mathrm{H}$, Yeung HW, Gonen $\mathrm{M}$, et al., Head and neck cancer: clinical userfulness and accuracy of PET/CT image fusion,
Radiology, 2004:231:65-72

10. Branstetter BF 4th, Blodgett TM, Zimmer LA, et al., Head and neck malignancy: is PET/CT more accurate than PET or CT alone?, Radiology, 2005;235:580-6.

11. Malone JP, Gerberi MA, Vasireddy S, et al., Early prediction of response to chemotherapy for head and neck cancer: reliability of restaging with combined positron emission tomography and computed tomography, Arch Otolaryngol Head Neck Surg. 2009:135:1119-25.

12 Chen AY, Vilaseca I, Hudgins PA, et al., PET-CT vs contrastenhanced CT: what is the role for each after chemoradiation for advanced oropharyngeal cancer?, Head Neck, 2006;28:487-95.

13. Isles MG, McConkey C, Mehanna HM, A systematic review and meta-analysis of the role of positron emission tomography in the follow up of head and neck squamous cell carcinoma following radiotherapy or chemoradiotherapy, Clin Otolaryngol, 2008;33:210-22.

14. Frank DK, Hu KS, Culliney BE, et al., Planned neck dissection after concomitant radiochemotherapy for advanced head and neck cancer, Laryngoscope, 2005;115:1015-20.

15. Stenson KM, Haraf DJ, Pelzer $H$, et al., The role of cervical lyphadenectomy after aggressive concomitant chemoradiotherapy: the feasibility of selective neck dissection, Arch Otolaryngol Head Neck Surg, 2000;126:950-6.

16. Brizel DM, Prosnitz RG, Hunter S, et al., Necesity for adjuvant neck dissection in setting of current chemoradiation for advanced head-and-neck cancer, Int I Radiat Oncol Biol Phys, 2004;58:1418-23

17. Nayak JV, Walvekar RR, Andrade RS, et al., Deferring planned neck dissection following chemoradiation for stage IV head and neck cancer: the utility of PET-CT, Laryngoscope, 2007;117:2129-34

18. Yao M, Graham MM, Hoffman HT, et al., The role of postradiation therapy FDG-PET in prediction of necessity of postradiation therapy neck dissection in locally advanced headand-neck squamous cell carcinoma, Int J Radiat Oncol Biol Phys, 2004:59:1001-10.

19. Goguen LA, Posner MR, Tishler RB, et al., Examining the need for neck dissection in the era of chemoradiation therapy for advanced head and neck cancer, Arch Otolaryngol Head Neck Surg, 2006;132:526-31.

20. Wang YF, Liu RS, Chu PY, et al., Positron emission tomography in surveillance of head and neck squamous cell carcinoma after difinitive chemoradiotherapy, Head Neck, 2009;31:442-51.

21. Gourin $C G$, Williams HT, Seabolt WN, et al., Utility of positron emission tomography-computed tomography in identification of residual disease after chemoradiation for advanced head and neck cancer, Laryngoscope, 2006;116:705-10.

22. Goerres GW, Schmid DT, Bandhauer F, et al., Positron emission tomography in the early follow-up of advanced head and neck cancer, Arch Otolaryngol Head Neck Surg, 2004;130:105-9.

23. Rabalais AG, Walvekar R, Nuss $D$, et al., Positron emission tomography-computed tomography surveillance for the nodepositive neck after chemoradiography, Laryngoscope, 2009;119:1120-4.

24. Malone J, Robbins KT, Neck dissection after chemoradiation for carcinoma of the upper aerodigestive tract: indications and complications, Curr Opin Otolaryngol Head Neck Surg, 2010;18(2):89-94. 